\title{
Unique separator-spectrometer experiments at the frontiers of nuclear physics: the Super-FRS scientific program
}

\author{
Christoph Scheidenberger ${ }^{1,2}$, Sydney Gales ${ }^{3,4}$, Hans Geissel ${ }^{1,2}$, Haik Simon ${ }^{1}$, Isao Tanihata ${ }^{5,6}$, and \\ Martin Winkler ${ }^{1}$ for the Super-FRS Collaboration \\ ${ }^{1}$ GSI Helmholtzzentrum für Schwerionenforschung, Darmstadt, Germany \\ ${ }^{2}$ II. Physikalisches Institut, Justus-Liebig-Universität Gießen, Gießen, Germany \\ ${ }^{3}$ ELI-NP@Horia Hulubei, National Institute for Physics and Nucl. Engineering, Măgurele, Romania \\ ${ }^{4}$ IPN Orsay/IN2P3-CNRS and University Paris XI, Orsay, France \\ ${ }^{5}$ School of Physics and Nucl. Energy Engineering and IRCNPC, Beihang University, Beijing, China \\ ${ }^{6}$ Research Center for Nuclear Physics, Osaka University, Osaka, Japan
}

\begin{abstract}
The superconducting fragment separator (Super-FRS) is the magnetic highresolution spectrometer, which will be coupled to the heavy-ion synchrotron complex at the future Facility for Antiproton and Ion Research FAIR. It will enable a variety of unique nuclear physics experiments. Key examples of the experimental program are presented, for instance the production and study of exotic hypernuclei (i.e.: nuclei far-off stability containing hyperons), the production and study of mesic atoms (i.e.: atoms containing bound mesons, like pions or eta mesons), the discovery of new neutron-rich isotopes, the search for new phenomena in weakly bound or dilute nuclear systems, and the search for neutron radioactivity, an elementary radioactive decay mode, which has not been discovered yet.
\end{abstract}

\section{The Super-FRS, a multi-stage high-resolution separator-spectrometer}

In this contribution an experimental program is sketched, which takes advantage of the nature of the Super-FRS [1] being a high-resolution separator-spectrometer. In general, the Super-FRS will be the central device of the NuSTAR collaboration at FAIR. With intense primary beams ranging from hydrogen to uranium in the energy range up to $1,500 \mathrm{~A} \mathrm{MeV}$, universal isotope production mechanisms (fragmentation, fission, spallation) and in-flight separation at a maximum magnetic rigidity of $20 \mathrm{Tm}$, it will provide several thousand short-lived isotopes for the detector systems located at the exits of its three branches [2]: HISPEC/DESPEC and MATS/LaSPEC at the Low-Energy Branch (LEB), R3B at the High-Energy Branch and ILIMA at the Storage-Ring Branch; these are the approved experiments within the modularized start version MSV of FAIR. For these detector systems, the Super-FRS will deliver mono-isotopic beams or cocktail beams of exotic nuclei over the entire chart of nuclei up to ${ }^{238} \mathrm{U}$; they will allow to study nuclear properties and dynamics in great detail. In order to reach mono-isotopic separation for secondary beams, which are characterized by a large phase space (typically around $40 \pi \mathrm{m} \mathrm{mrad}$ ) and mean velocities up to $\sim 95 \%$ of the speed of light, a momentum resolving power of the order of $\mathrm{p} / \Delta \mathrm{p} \sim 1,500$ is required. It is accomplished with a multistage magnetic system (first and second half of the pre-separator, first and second half of the mainseparator), which comprises degrader stations at the central foci of pre- and main-separator (see 
figure 1). The so-called $\mathrm{B} \rho-\Delta \mathrm{E}-\mathrm{B} \rho$ separation scheme ( $\mathrm{B} \rho$ stands for magnetic rigidity analysis, followed by the energy loss $\Delta \mathrm{E}$ in a degrader and subsequent $\mathrm{B} \rho$-analysis, see ref. [1]) leads to the suppression of the primary beam and unwanted secondary species; with simultaneous momentum measurements by particle tracking using specialized detector systems (for position, angle, time-offlight and energy deposition) unambiguous particle identification is achieved and highest selectivity is reached, e.g. one secondary ion can be singled-out from some $10^{15}$ primary beam ions.

The experiments mentioned above will study the in-flight separated exotic nuclei by placing the detector systems at the three exits of the Super-FRS, while the present contribution describes experiments, which will use the separator-spectrometer capabilities as such. A key feature common to all of them is the high-resolution momentum measurement. The highest possible momentum resolution will be reached with the energy-buncher/spectrometer [3] at the LEB when this is coupled to the main separator in a dispersion-matched ion-optical mode: to first order, this combination will yield a momentum resolving power up to $\mathrm{p} / \Delta \mathrm{p} \sim 20,000$. Another important trait is the multi-stage operation (see next section). With these features, inherent to the system, a variety of scientifically appealing experiments will become possible with the Super-FRS separator-spectrometer and the LEB buncher-spectrometer as such. These are sketched below.

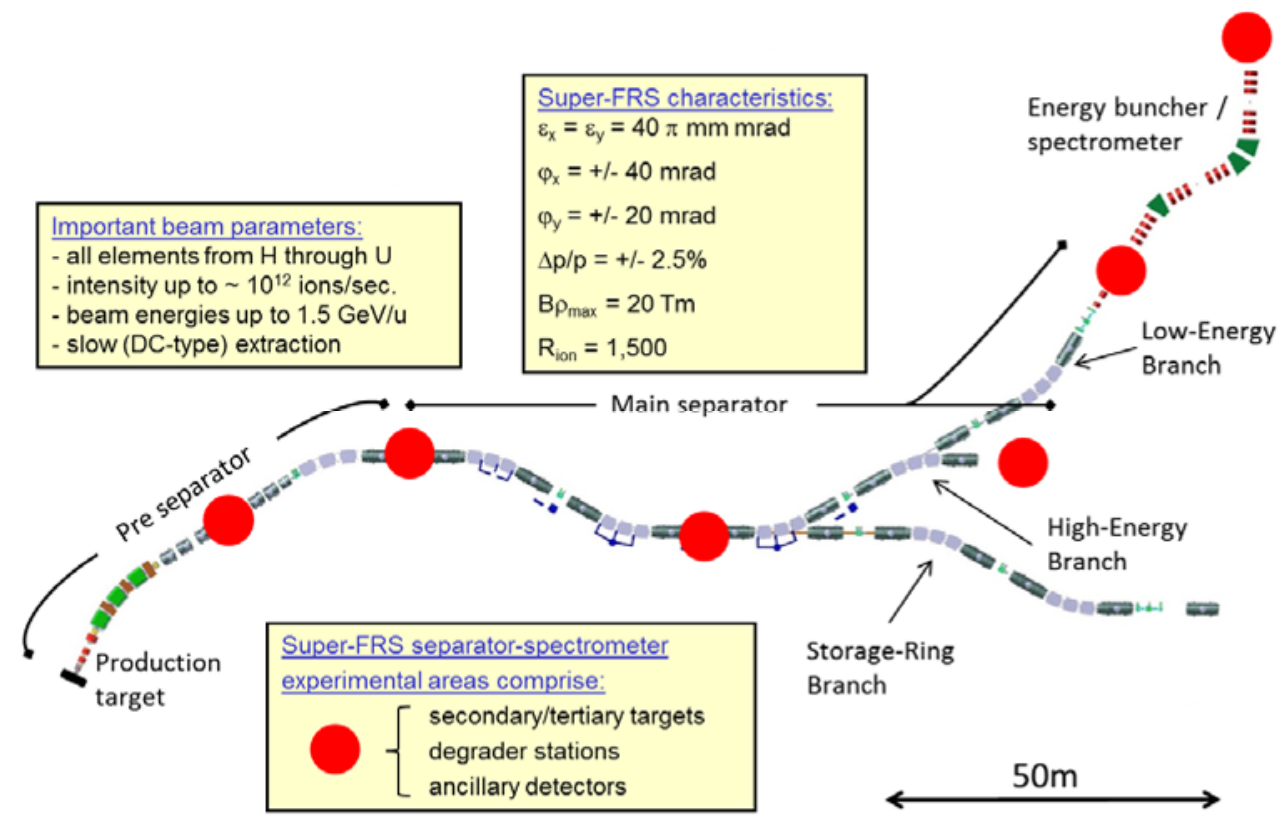

Figure 1. Schematic view and characteristic features of the Super-FRS with its three branches and the LEB energy-buncher/spectrometer system. The different separator stages and the experimental areas, which will be used for high-resolution separator-spectrometer experiments, are indicated.

\section{Planned separator-spectrometer experiments}

The headline of the planned experiments is that they will exploit the separator-spectrometer capabilities as such, respectively as a comprising and integral part of the measurement. With this intention in mind, the Super-FRS Collaboration has figured out experiments, which a) are complementary to the other NuSTAR experiments mentioned above, b) are intrinsically connected with the original purpose of the facility (production and separation of exotic nuclei, including new isotopes), c) are unique or d) emerge as new physics opportunities with this instrument. The common feature is that they will employ

- individual stages of the Super-FRS (roughly speaking: pre-separator, first and second half of the main separator, spectrometer/energy buncher at the Low-Energy Branch) 
- different ion-optical modes of the Super-FRS (achromatic, mono-energetic, dispersive)

- several target and degrader stations at the major focal planes (including secondary or tertiary ${ }^{\mathrm{a}}$ targets)

- various functions (separation including the suppression of the primary beam or unwanted species, identification, momentum measurement).

These items can be used in individual combinations, customized for the specific goals of the measurement. This opens up a large variety of modes and experimental conditions, including new measurement concepts, not fully explored yet. For most of the experiments, the standard equipment of the Super-FRS can be used, while some experiments will need additional ancillary detector setups, which are currently under development. Scientific contributions to the following main topics can be expected:

Basic studies: this category of Super-FRS experiments goes hand in hand with the performance verification of the separator and its standard detector systems; it will yield first physics results during the commissioning phase. Ion-optical measurements will also include atomic interaction experiments of ions penetrating (shaped) matter over the full energy range down to thermalized reaction products [4] (e.g. in the cryogenic gas-filled stopping cell of the Low-Energy Branch [5]). These early studies are unique and address also important frontiers of nuclear physics such as the search for new isotopes [6], production cross section and rate measurements and exploratory mass and half-life measurements. Inclusive gross property studies will provide new basic data needed for all other NuSTAR experiments, which have dedicated setups (mentioned above) at the three exit branches of the Super-FRS for detailed and/or more exclusive and/or kinematically complete measurements. Furthermore, exotic nuclei or exotic atoms (see below) can be produced in one of the first stages of the Super-FRS and their properties can be investigated with the high momentum resolution in one of the subsequent ion-optical stages operated in the dispersion-matched mode. This will give access to nuclear radii and matter distributions of the most exotic nuclei.

Nuclear structure: the structure of nuclei far off stability is a major topic of modern nuclear physics and a central pillar of all next-generation radioactive beam facilities. While there are several different spectroscopic approaches, new experimental techniques are associated with nuclear reactions at relativistic speed and subsequent high-momentum resolution spectroscopy. For instance total interaction and nuclear charge-changing cross section measurements, followed by longitudinal momentum-distribution measurements, introduced a new view at nuclei: the discoveries of neutron and proton halos, neutron skins and new „magic numbers“ [7] originated from such inclusive experiments at high-resolution magnetic spectrometers. They benefit from thick targets and can be performed with rates as low as one particle per second. Exotic decay modes, such as 2-proton radioactivity [8], appear near the drip-lines at the limits of stability and are subject of particular interest. Neutron radioactivity is hitherto unobserved. Because neutrons experience only the centrifugal barrier, pairing correlations between neutrons in nuclei may have an important influence on the lifetime and can potentially be studied [9]. Multiple neutron or proton radioactivity, appearing in extremely short-lived nuclei $\left(\mathrm{T}_{1 / 2} \sim 10^{-10} \mathrm{~s}\right)$, can only be studied with the in-flight decay technique, which was pioneered at the FRS [10], and which is based on relativistic exotic nuclei impinging on a secondary target within a fragment separator and subsequent identification. Also beta-delayed ternary fission is so far unobserved, but with the sensitivity of novel detector systems, such as an O-TPC [11], combined with the universal isotope production capabilities, short separation times and the selectivity of Super-FRS, even very weak decay branches can be identified. An example is the very weak betadelayed 3p-decay branch in ${ }^{31} \operatorname{Ar}[12]$, which was recently observed with an O-TPC at the FRS. Finally, the study of tensor forces is an important new direction in nuclear physics [13]. The tensor

\footnotetext{
a Several scenarios for the use of tertiary targets can be considered; examples are a) reaction studies at the energybuncher/spectrometer, where very exotic nuclei -produced in so-called „two-step reactions“ in a primary and secondary target, located at the entrance of the pre-separator and the central focal plane of the main separator, respectively- undergo nuclear reactions in a tertiary target located at the entrance of the energy-buncher/spectrometer, or b) charge-changing and total interaction cross section measurements of fragment-like ejectiles from secondary reactions at the central focal plane of the main separator.
} 
interaction introduces a high-momentum component in the nucleus through specific proton-neutron correlations, which can be "visualized" by high-momentum transfer reactions and subsequent highresolution momentum analysis of the beam-like reaction product at beam energies above $400 \mathrm{~A} \mathrm{MeV}$. The new data, expected in the future, will provide important information for the development of theories or models which go beyond the present shell model.

Nuclear astrophysics and cosmo-nuclear physics: to this wide and interdisciplinary field, the collaboration may contribute two important pieces: one is the study of the equation-of-state (EOS) of cold asymmetric matter by nuclear density distributions and neutron skin thicknesses, where the saturation density of asymmetric nuclear matter can be extracted from systematic data on interaction cross sections, charge changing cross sections, proton elastic scattering of neutron rich nuclei in an "active target"; the other property of EOS, nuclear compressibility, can be studied from isoscalar giant monopole resonances, which can efficiently be excited by inelastic scattering of relativistic exotic nuclei off alpha particles. Also the nuclear gross property and structure data mentioned above, especially the properties of the r-process nuclei, will provide important experimental input data for understanding the stellar and explosive nucleosynthesis path ways.

Exotic atoms: the integration of particles other than protons and neutrons in a nucleus or other than electrons in its atomic shells is an important method to probe basic features of the strong interaction or to test nuclear theory. At relativistic energies and with sufficiently intense beams (typically in excess of $\sim 10^{5}$ ions per second), various exotic atoms can be produced, for instance hypernuclei or $\Delta$ and $\mathrm{N}^{*}$ excitations in nuclei or mesic nuclei respectively mesic atoms. Both are of interest to study chiral symmetry breaking via direct measurements of in-medium mass modifications [14]. Also interactions between such exotic particles and nucleons or nuclear matter are subjects of interest; it will be a forthcoming study whether and how these can be studied experimentally. Resonance physics in radioactive nuclei is completely unexplored. A recent pilot experiment [15] with the FRS has demonstrated that new properties of the $\Delta$ resonances can be observed in charge-exchange reactions of heavy ions. Also the possibility to produce (multiple) strangeness in nuclei by coalescence in fragmentation reactions, recently demonstrated [16], opens up a completely new and wide field of studies: the third dimension of the chart of nuclei. Production rates for exotic atoms are sufficiently high in the region of $1 \ldots 1.5 \mathrm{~A} \mathrm{GeV}$ (cross sections are at the micro-barn level), and the Super-FRS with its capability to serve as zero-degree spectrometer, its momentum resolution down to $\delta \mathrm{p} \sim 10^{-4}$ (essential for missing-mass measurements of bound states with a precision of $\sim 1 \mathrm{MeV} / \mathrm{c}^{2}$ ), and its high primary-beam or contaminant suppression will allow unique experiments in this emerging field.

\section{Conclusion}

The Super-FRS separator-spectrometer will enable new physics opportunities, which will be unique and characteristic for FAIR, scientifically appealing, and exhibit a long-term scientific perspective. In the future, even more new ideas, realizable with this versatile instrument, may come up.

\section{Acknowledgements}

Fruitful discussions with the following colleagues are gratefully acknowledged: J. Aysto, K.-H. Behr, J. Benlliure, A. Bracco, P. Egelhof, A. Fomichev, M. Harakeh, R. Hayano, S. Heinz, A. Herlert, K. Itahashi, A. Jokinen, N. Kalantar, R. Kanungo, D. Krämer, H. Lenske, J. Meng, V. Metag, I. Mukha, G. Münzenberg, C. Nociforo, J. Ong, M. Pfützner, A. Prochazka, S. Pietri, Y.L.Pivovarov, W.R. Plaß, S. Puroshothaman, C. Rappold, T. Saito, H. Toki, B. Voss, H. Weick, J. Winfield, V. Zamfir.

\section{References}

1. H. Geissel et al., Nucl. Instr. Meth. B204, 71 (2003).

2. FAIR Baseline Technical Report (2006), see 
http://www.fair-center.de/fileadmin/fair/publications FAIR/FAIR_GreenPaper 2009.pdf

3. J. Winfield et al., Nucl. Instr. Meth. A704, 76 (2013).

4. H. Geissel et al., Nucl. Instr. Meth. B195, 3 (2002).

5. W. R. Plaß et al., Nucl. Instr. Meth. B (2013), in press.

6. J. Kurcewicz et al., Phys. Lett. B717, 371 (2012).

7. I. Tanihata et al., Phys. Rev. Lett. 55, 2676 (1985), and Phys. Lett. B206, 592 (1988);

W. Schwab et al., Z. Phys. A350, 283 (1995);

T. Suzuki et al., Phys. Rev. Lett. 75, 3241 (1995);

A. Ozawa et al., Phys. Rev. Lett. 84, 5493 (2000).

8. M. Pfützner et al. Eur. Phys. J. A14, 279 (2002).

9. L. Grigorenko et al., Phys. Rev. C84, 021303 (2011).

10. I. Mukha et al., Phys. Rev. Lett. 99, 182501 (2007).

11. M. Ćwiok et al, IEEE TNS 52, 2895 (2005), K.Miernik et al., Nucl.Instr.Meth. A581, 194 (2007).

12. M. Pfützner et al., publication in preparation.

13. H. J. Ong et al., Phys. Lett. B725, 277(2013).

14. T. Yamazaki et al., Z. Phys. A355, 219 (1996);

K. Itahashi et al., Prog. Theor. Phys. 128, 601 (2012).

15. J. Benlliure et al., publication in preparation.

16. C. Rappold et al., Nucl. Phys. A 913, 170 (2013). 
